\title{
Evidências adicionais sobre a sazonalidade dos retornos diários do mercado de ações brasileiro
}

\author{
André Assis de Salles (UFRJ) asalles@ind.ufrj.br
}

\begin{abstract}
Resumo
A sazonalidade dos retornos de ativos financeiros tem sido freqüentemente estudada por intermédio de anomalias de mercado designadas como efeito calendário, que pode ser definido como a tendência dos retornos a apresentar desempenho diferenciado em determinado período de tempo: no caso do efeito dia da semana, associado ao dia da semana, e no caso de efeito fim de semana, associado a retornos diferenciados na segundafeira e na sexta-feira. Este trabalho, a partir de informações de retornos do mercado de ações brasileiro, procura verificar a existência de retornos diferenciados nos dias da semana, através de modelos autoregressivos heterocedásticos. Esses modelos foram desenvolvidos com um enfoque Bayesiano, utilizando-se Monte Carlo Markov Chain via amostrador de Gibbs, para dados distribuidos normalmente e distribuidos de acordo com a distribuição t de Student.
\end{abstract}

Palavras chave: Monte Carlo Markov Chain; Efeito Dia da Semana; Eficiência de Mercado.

\section{Introdução}

Dentre as anomalias do mercado de capitais, designadas como efeito calendário, o efeito dia da semana e o efeito janeiro têm sido freqüentemente pesquisados. $O$ tema atrai grande interesse dos agentes econômicos envolvidos no desenvolvimento de estratégias de negociação nos mercados financeiros, uma vez que permitem a visualização de imperfeições do mercado, ou de sinais de diminuição da eficiência informacional do mercado. Assim o estudo do tema, além de permitir um conhecimento mais profundo do comportamento da série temporal de retornos dos ativos financeiros, tem importância, no estudo de finanças, pois a existência da sazonalidade desses retornos é totalmente incompatível com a hipótese de eficiência de mercado, importante axioma da teoria de finanças. Os estudos sobre sazonalidade dos retornos de ativos financeiros têm sido elaborados, em geral, por meio dessas anomalias de mercado, ou seja ineficiências associadas ao calendário - efeito calendário, que pode ser definido como a tendência dos retornos a apresentar desempenho diferenciado, de forma sistemática, em determinado período de tempo: no caso do efeito dia da semana, associado ao dia da semana ou aos retornos anormais em determinado dia da semana, e no caso de efeito fim de semana, associado a retornos diferenciados na segundafeira e na sexta-feira ou aos retornos anormais na segunda-feira e na sexta-feira.

Os trabalhos pioneiros sobre o assunto tiveram seus resultados apresentados na década de 70 e início da década de 80. Dentre esses trabalhos devem ser mencionados o de CROSS (1973), o de FRENCH (1980) e o de GIBBONS \& HESS (1981). A partir da publicação desses trabalhos, as pesquisas têm se intensificado: tanto no mercado norte-americano de ações como em outros mercados, desenvolvidos e emergentes. Entretanto os efeitos calendários podem não se manter no tempo, o que tem motivado a revisão de tópicos relacionados ao tema. Assim, na última década têm surgido novas pesquisas sobre efeito dia da semana, do efeito fim de semana, e outras de anomalias com o intuito de confirmar, ou questionar, os resultados obtidos no passado (ver AGRAWAL \& TANDON (1994)). Em pesquisa com informações de retornos de cinco mercados asiáticos, para o período de dezembro de 1989 a janeiro de 1996, 
BROOKS \& PERSAND (2001) não encontraram sinais do efeito dia da semana nos mercados acionários da Coréia do Sul e das Filipinas, e não verificam um padrão para os efeitos detectados nos outros mercados estudados: Tailândia, Malásia e Taiwan. Nesse trabalho BROOKS \& PERSAND (2001) estudam, também, a sazonalidade do risco de mercado. Algumas pesquisas mais recentes como a de e de LIN \& LIM (2001), com dados do mercado australiano de ações, e a de KOHERS et al. (2004), com dados dos maiores mercados de ações internacionais, têm mostrado o desaparecimento, ou diminuição com o tempo, do efeito dia da semana, o que se constitui em um indicador de aumento da eficiência informacional dos mercados de ações. Em trabalho divulgado recentemente BASHER \& SADORSKY (2005), a partir de uma amostra abrangente com informações dos retornos das cotações diárias de índices de lucratividade do mercado de ações de 21 países emergentes, verificam o efeito dia da semana nesses países através de modelos clássicos para detectar a sazonalidade diária dos retornos, assim como do risco de mercado desses países, utilizando dentre outros o modelo para verificação do risco de mercado apresentado na pesquisa realizada por BROOKS \& PERSAND (2001). Os resultados obtidos não apontam para existência do efeito dia da semana na maioria dos mercados estudados. Por outro lado no que se refere ao risco de mercado os resultados apontaram para sazonalidade, ou seja, efeito dia da semana para risco sistemático. Com dados sobre o mercado brasileiro foram realizados poucos trabalhos dentre os mais recentes pode-se citar o de TORRES et al. (2000), que utilizando métodos clássicos não rejeita a hipótese de retornos diferenciados na segunda feira.

Além da variação dos efeitos calendário no tempo, o desenvolvimento e métodos econométricos têm motivado a continuidade da feitura de pesquisas sobre tópicos relacionados aos efeitos calendário. O grande desenvolvimento, nas últimas décadas, de procedimentos utilizados na inferência estatística, em particular da inferência Bayesiana tem permitido pesquisas mais recentes utilizando sofisticados métodos econométricos, desenvolvidos durante as últimas décadas, possibilitando a elaboração de modelos heterocedásticos, de importância extrema para a modelagem de séries de retornos de ativos financeiros. Atualmente estão disponíveis técnicas, e procedimentos, que podem vir a permitir melhores inferências acerca das anomalias, designadas na literatura de finanças por efeitos calendário. $\mathrm{Na}$ elaboração deste trabalho são utilizadas algumas dessas técnicas, e procedimentos, no estudo do efeito dia da semana nos retornos do mercado de ações brasileiro em períodos anuais da última década.

As seções seguintes tratam, respectivamente: dos objetivos do trabalho, na seção 2; da abordagem metodológica, na seção 3; dos dados utilizados, na seção 4; da análise dos resultados obtidos, na quinta seção; e das considerações finais, na sexta e última seção.

\section{Objetivos}

Este trabalho tem por objetivos: estudar, utilizando procedimentos da inferência Bayesiana, o efeito dia da semana e o efeito fim de semana no mercado acionário brasileiro, a partir de retornos diários em dólares, do Ibovespa, principal índice de lucratividade da Bolsa de Valores de São de Paulo (BOVESPA) que representa a quase totalidade dos negócios com ações no Brasil; e verificar a existência de variações desses efeitos no tempo, ano a ano, por intermédio de amostras de retornos diários do Ibovespa, de abril 1994 a dezembro de 2004.

\section{Abordagem metodológica}

Como observado na introdução deste trabalho, o problema da heterocedasticidade das séries de retornos das cotações de ativos financeiros não pode ser ignorado. Um modelo para comportamento dos retornos do índice Ibovespa que considere esse problema deve ser utilizado de forma a se atingir melhores resultados, ao se testar a hipótese dos retornos do mercado acionário brasileiro não apresentar variações ocasionadas por negociações ocorridas 
em determinado dia da semana. Assim, para representar realizações de processos estocásticos aqui estudados, foram desenvolvidos modelos autoregressivos heterocedásticos com variância dos retornos seguindo um modelo Exponentially Weighted Moving Average (EWMA) em quatro formatos para média dos retornos: primeiro um modelo autoregressivo para a média; segundo um modelo autoregressivo para média, acrescido de uma variável binária representando o retorno das segundas-feiras; terceiro um modelo autoregressivo para média, acrescido de cinco variáveis binárias representando, respectivamente, os retornos de cada um dos dias da semana; e quarto, um modelo que se diferencia do anterior, o terceiro, por não conter o intercepto. A escolha do modelo EWMA, em detrimento de outros modelos de volatilidade disponíveis, foi feita pela maior rapidez na obtenção dos resultados das simulações estocásticas e por sua utilização entre as instituições atuantes no mercado de capitais. Em todos os modelos foi assumida a normalidade dos retornos e, alternativamente, a distribuição $t$ de Student para a distribuição dos retornos diários o mercado acionário. Pois algumas pesquisas têm indicado que os retornos diários dos índices de ações dos mercados internacionais se aproximam de uma distribuição $t$ de Student com um número de graus de liberdade em torno de 3, como observado por PEIRÓ (1994). Assim para todos os modelos normais foram elaboradas alternativas com a distribuição t de Student, com o número de graus de liberdade determinado no modelo. Assim foram desenvolvidos modelos Bayesianos e na obtenção de cada distribuição posteriori dos parâmetros de interesse, para o processo de inferência, foram utilizados métodos numéricos baseados em Monte Carlo Markov Chain. Os modelos desenvolvidos foram implementados no software BUGS (Bayesian Inference Using Gibbs Sampler), na versão WinBUGS 1.4, elaborado por SPIEGELHALTER et al. (2003), para determinação das posterioris dos parâmetros dos modelos utilizando Monte Carlo Markov Chain via amostrador de Gibbs. Para um melhor entendimento dos procedimentos da inferência Bayesiana utilizados neste trabalho pode-se recorrer a MIGON \& GAMERMAN (1999). Enquanto para um maior conhecimento, ou aprofundamento, das técnicas de Monte Carlo Markov Chain (MCMC) para inferência Bayesiana pode-se recorrer a GAMERMAN (1997) e GILKS et al. (1998).

Os modelos autoregressivos heterocedásticos utilizados neste trabalho, designados adiante por AR(1) - EWMA, podem ser descritos da seguinte forma:

- modelo 1

$$
\begin{aligned}
& \left(R_{t} \mid I_{t-1}\right) \sim \operatorname{Normal}\left(\mu_{t} ; \sigma_{t}^{2}\right) \\
& \mu_{t}=a+b R_{t-1} \\
& \sigma_{t}^{2}=\lambda \sigma_{t-1}^{2}+(1-\lambda) R_{t-1}^{2}
\end{aligned}
$$$$
\left(R_{t} \mid I_{t-1}\right) \sim \operatorname{Student}\left(\mu_{t} ; \sigma_{t}^{2}, v\right)
$$

$\mu_{t}=a+b R_{t-1}$

$\sigma_{t}^{2}=\lambda \sigma_{t-1}^{2}+(1-\lambda) R_{t-1}^{2}$

- modelo 2

$$
\begin{aligned}
& \left(R_{t} \mid I_{t-1}\right) \sim \operatorname{Normal}\left(\mu_{t} ; \sigma_{t}^{2}\right) \\
& \mu_{t}=a+b R_{t-1}+c_{1} D_{1} \\
& \sigma_{t}^{2}=\lambda \sigma_{t-1}^{2}+(1-\lambda) R_{t-1}^{2}
\end{aligned}
$$

$\mathrm{ou}$

$$
\begin{aligned}
& \left(R_{t} \mid I_{t-1}\right) \sim \operatorname{Student}\left(\mu_{t} ; \sigma_{t}^{2}, v\right) \\
& \mu_{t}=a+b R_{t-1}+c_{1} D_{1} \\
& \sigma_{t}^{2}=\lambda \sigma_{t-1}^{2}+(1-\lambda) R_{t-1}^{2}
\end{aligned}
$$

- modelo 3

$$
\begin{aligned}
& \left(R_{t} \mid I_{t-1}\right) \sim \operatorname{Normal}\left(\mu_{t} ; \sigma_{t}^{2}\right) \\
& \mu_{t}=a+b R_{t-1}+\sum_{i=1}^{5} c_{i} D_{i} \\
& \sigma_{t}^{2}=\lambda \sigma_{t-1}^{2}+(1-\lambda) R_{t-1}^{2}
\end{aligned}
$$

$$
\begin{aligned}
& \left(R_{t} \mid I_{t-1}\right) \sim \operatorname{Student}\left(\mu_{t} ; \sigma_{t}^{2}, v\right) \\
& \mu_{t}=a+b R_{t-1}+\sum_{i=1}^{5} c_{i} D_{i} \\
& \sigma_{t}^{2}=\lambda \sigma_{t-1}^{2}+(1-\lambda) R_{t-1}^{2}
\end{aligned}
$$


- modelo 4

$$
\begin{aligned}
& \left(R_{t} \mid I_{t-1}\right) \sim \operatorname{Student}\left(\mu_{t} ; \sigma_{t}^{2}, v\right) \\
& \mu_{t}=b R_{t-1}+\sum_{i=1}^{5} c_{i} D_{i} \\
& \sigma_{t}^{2}=\lambda \sigma_{t-1}^{2}+(1-\lambda) R_{t-1}^{2}
\end{aligned}
$$

onde,

$R_{t}=$ retorno da cotação de fechamento do índice Ibovespa,

$I_{t}=$ informações disponíveis até o período $t$,

$D_{t}=$ variável dummy no período $t$,

$\mu_{t}=$ média da distribuição dos retornos no período $t, \mathrm{e}$

$\sigma_{t}^{2}=$ variância dos retornos no período $t$.

Após realização das simulações com os modelos AR(1)-EWMA, descritos anteriormente, procedeu-se a comparação desses modelos com vistas a seleção do mais adequado para ser aplicado aos dados das subamostras anuais dos retornos diários do Ibovespa. O critério utilizado, na seleção desse modelo, foi uma generalização do critério de Akaike, o AIC (Akaike Information Criterion), proposta por SPIEGELHALTER et al. (2002). O critério DIC (Deviance Information Criterion) que está implementado na versão do software BUGS utilizada para este trabalho. Desta forma o modelo selecionado deve maximizar o DIC obtido nas simulações realizada no WinBUGS para determinação das posterioris dos parâmetros de interesse. Para implementação dos modelos foram utilizadas as informações descritas a seguir.

\section{Amostra - dados utilizados}

Para este trabalho foram coletadas cotações de fechamento em dólares norte-americanos do índice de lucratividade da Bolsa de Valores de São Paulo (BOVESPA), o Ibovespa, para o período de abril de 1994 até dezembro de 2004. A partir dessas cotações foram calculados retornos diários da seguinte forma:

$$
R_{t}=\ln \left(\frac{\text { Ibovespa }_{t}}{\text { Ibovespa }_{t-1}}\right)
$$

Os resultados obtidos, a partir desses dados, são descritos detalhadamente na seção seguinte.

\section{Análise dos resultados obtidos}

Os resultados obtidos com cada um dos modelos autoregressivos AR(1) - EWMA sugeridos, após 11 mil iterações descartadas as primeiras mil, estão listados na tabela 1 adiante: as informações se referem as médias da posteriori de cada parâmetro, e entre parênteses o respectivo desvio padrão. A amostra utilizada para obtenção dos resultados apresentados na tabela 1 corresponde aos retornos diários de 2 de janeiro a 11 de dezembro de 2002, perfazendo 232 observações. Os resultados contemplam os modelos que supõem distribuição normal e $\mathrm{t}$ de Student para os retornos do Ibovespa. Da tabela 1, no que se refere aos resultados do modelo 1 para dados de retornos do índice Ibovespa, observa-se que os dados se ajustam bem ao modelo AR(1)-EWMA, com o pressuposto de normalidade dos retornos ou com a suposição dos retornos se ajustarem a distribuição $t$ de Student com oito graus de liberdade. Os resultados que se referem ao modelo 2, permitem inferir se existem retornos diferenciados em média nas segundas-feiras. O bom ajuste dos modelos e a significância estatística dos parâmetros, com exceção da constante quando se trata dos retornos do Ibovespa, apontam que a hipótese de retornos diferenciados nas segundas-feiras não pode ser 
descartada para o Ibovespa, isto é, a hipótese de existência do efeito fim de semana não é rejeitada para os retornos diários do índice Ibovespa. Quanto ao número de graus de liberdade da distribuição $t$ de Student, determinada com a simulação do modelo: foi igual a 8 para simulações com o Ibovespa.

\begin{tabular}{|c|c|c|c|c|c|c|c|}
\hline \multirow{2}{*}{ Parâmetro } & \multicolumn{2}{|l|}{ Modelo 1} & \multicolumn{2}{|l|}{ Modelo 2} & \multicolumn{2}{|l|}{ Modelo 3} & \multirow{2}{*}{$\begin{array}{l}\text { Modelo } 4 \\
\text { Student }\end{array}$} \\
\hline & Normal & Student & Normal & Student & Normal & Student & \\
\hline$a$ & $\begin{array}{l}-0,0032 \\
(0,0015)\end{array}$ & $\begin{array}{l}-0,0030 \\
(0,0016)\end{array}$ & $\begin{array}{l}-0,0017 \\
(0,0017)\end{array}$ & $\begin{array}{l}-0,0011 \\
(0,0015)\end{array}$ & $\begin{array}{l}-0,0040 \\
(0,0024)\end{array}$ & $\begin{array}{l}-0,0023 \\
(0,0027)\end{array}$ & -- \\
\hline$b$ & $\begin{array}{c}0,1843 \\
(0,0685)\end{array}$ & $\begin{array}{c}0,1899 \\
(0,0714)\end{array}$ & $\begin{array}{c}0,1893 \\
(0,0682)\end{array}$ & $\begin{array}{c}0,2670 \\
(0,0736)\end{array}$ & $\begin{array}{c}0,1963 \\
(0,0696)\end{array}$ & $\begin{array}{c}0,1939 \\
(0,0732)\end{array}$ & $\begin{array}{c}0,2056 \\
(0,0743)\end{array}$ \\
\hline $\begin{array}{c}c_{1} \\
(\operatorname{segunda})\end{array}$ & -- & -- & $\begin{array}{l}-0,0072 \\
(0,0037)\end{array}$ & $\begin{array}{c}-0,0034 \\
(0,0031)\end{array}$ & $\begin{array}{l}-0,0049 \\
(0,0042)\end{array}$ & $\begin{array}{l}-0,0077 \\
(0,0046)\end{array}$ & $\begin{array}{c}-0,0092 \\
(0,0038)\end{array}$ \\
\hline $\begin{array}{c}c_{2} \\
(\operatorname{terça})\end{array}$ & -- & -- & -- & -- & $\begin{array}{c}0,0014 \\
(0,0042)\end{array}$ & $\begin{array}{l}-0,0010 \\
(0,0046)\end{array}$ & $\begin{array}{c}-0,0037 \\
(0,0037)\end{array}$ \\
\hline $\begin{array}{c}c_{3} \\
(\text { quarta })\end{array}$ & -- & -- & -- & -- & $\begin{array}{c}-0,0078 \\
(0,0042)\end{array}$ & $\begin{array}{c}0,0056 \\
(0,0046)\end{array}$ & $\begin{array}{c}0,0034 \\
(0,0038)\end{array}$ \\
\hline $\begin{array}{c}c_{4} \\
(\text { quinta) }\end{array}$ & -- & -- & -- & -- & $\begin{array}{c}0,9826 \\
(2,2470)\end{array}$ & $\begin{array}{c}1,0300 \\
(2,2130)\end{array}$ & $\begin{array}{c}-0,0037 \\
(0,0041)\end{array}$ \\
\hline $\begin{array}{c}c_{5} \\
(\operatorname{sexta})\end{array}$ & -- & -- & -- & -- & $\begin{array}{c}1,0350 \\
(2,2370)\end{array}$ & $\begin{array}{c}0,9708 \\
(2,2440)\end{array}$ & $\begin{array}{c}-0,0000 \\
(0,0036)\end{array}$ \\
\hline$\lambda$ & $\begin{array}{c}0,9199 \\
(0,0246)\end{array}$ & $\begin{array}{c}0,9032 \\
(0,0389)\end{array}$ & $\begin{array}{c}0,9172 \\
(0,0246)\end{array}$ & $\begin{array}{c}0,8651 \\
(0,0421)\end{array}$ & $\begin{array}{c}0,9104 \\
(0,0258)\end{array}$ & $\begin{array}{c}0,8971 \\
(0,0477)\end{array}$ & $\begin{array}{c}0,8880 \\
(0,0507)\end{array}$ \\
\hline DIC & $-1048,32$ & $-1039,62$ & $-1042,79$ & $-1096,47$ & $-1042,12$ & $-1036,40$ & $-1009,04$ \\
\hline MSE & 0,005 & 0,005 & 0,005 & 0,005 & 0,005 & 0,005 & 0,005 \\
\hline
\end{tabular}

Tabela 1 - Resultados dos modelos AR(1) - EWMA

Ainda da tabela 1, pode-se observar que o ajuste dos modelos não melhora quando se leva em consideração a existência de retornos diferenciados nos dias da semana. Os resultados para o modelo 3 não permitem se observar diferença em relação ao modelo 1 , no que se refere ao erro médio quadrático (MSE). Enquanto a observação das estimativas dos coeficientes das variáveis binárias não permite a rejeição da hipótese de não existência de retornos diários 
diferenciados devido a sazonalidade na distribuição de retornos nos dias semana, ou ao efeito dia da semana. Isto é, a média das posterioris dos parâmetros das variáveis dummies não permite a aceitação da hipótese de retornos diferenciados nos dias da semana. No entanto observa-se, exceto nos resultados do modelo 3 - pressupondo a distribuição $t$ de Student para o Ibovespa, que a hipótese de retorno médio diferenciado nas quartas-feiras não pode ser descartada para os retornos diários da amostra utilizada, isto é, existe indicação da necessidade de outras inferências. Para o modelo $t$ de Student o número de graus de liberdade estimado foi em média igual a 5. Os resultados obtidos com o modelo 2 apontam para necessidade de maiores investigações a hipótese da existência do efeito fim de semana, assim como os resultados obtidos com o modelo 3 mostram indícios de retornos diferenciados nas quartas-feiras. Enquanto o modelo 4 não apresenta evidências de retornos diferenciados nos dias da semana.

\begin{tabular}{|c|c|c|c|c|c|c|c|c|c|c|c|}
\hline Parâmetro & 1994 & 1995 & 1996 & 1997 & 1998 & $\begin{array}{c}\text { Ano } \\
1999\end{array}$ & 2000 & 2001 & 2002 & 2003 & 2004 \\
\hline$n$ & 185 & 245 & 249 & 248 & 246 & 246 & 248 & 246 & 249 & 250 & 249 \\
\hline$b$ & $\begin{array}{c}0,162 \\
(0,093)\end{array}$ & $\begin{array}{c}0,194 \\
(0,079)\end{array}$ & $\begin{array}{c}0,015 \\
(0,079)\end{array}$ & $\begin{array}{c}0,161 \\
(0,079)\end{array}$ & $\begin{array}{c}0,056 \\
(0,078)\end{array}$ & $\begin{array}{c}0,129 \\
(0,062)\end{array}$ & $\begin{array}{c}0,067 \\
(0,077)\end{array}$ & $\begin{array}{c}0,129 \\
(0,062)\end{array}$ & $\begin{array}{c}0,221 \\
(0,072)\end{array}$ & $\begin{array}{c}0,099 \\
(0,074)\end{array}$ & $\begin{array}{c}0,165 \\
(0,075)\end{array}$ \\
\hline $\begin{array}{c}c_{1} \\
\text { segunda }\end{array}$ & $\begin{array}{c}0,006 \\
(0,006)\end{array}$ & $\begin{array}{c}-0,005 \\
(0,004)\end{array}$ & $\begin{array}{c}0,001 \\
(0,002)\end{array}$ & $\begin{array}{c}0,005 \\
(0,003)\end{array}$ & $\begin{array}{c}-0,001 \\
(0,004)\end{array}$ & $\begin{array}{l}-0,001 \\
(0,004)\end{array}$ & $\begin{array}{c}-0,004 \\
(0,003)\end{array}$ & $\begin{array}{c}-0,001 \\
(0,004)\end{array}$ & $\begin{array}{c}-0,009 \\
(0,004)\end{array}$ & $\begin{array}{c}0,002 \\
(0,003)\end{array}$ & $\begin{array}{c}0,000 \\
(0,003)\end{array}$ \\
\hline $\begin{array}{c}c_{2} \\
\text { terça }\end{array}$ & $\begin{array}{c}-0,002 \\
(0,006)\end{array}$ & $\begin{array}{c}-0,001 \\
(0,003)\end{array}$ & $\begin{array}{c}0,003 \\
(0,002)\end{array}$ & $\begin{array}{c}0,009 \\
(0,003)\end{array}$ & $\begin{array}{c}0,003 \\
(0,004)\end{array}$ & $\begin{array}{l}-0,001 \\
(0,003)\end{array}$ & $\begin{array}{l}-0,003 \\
(0,003)\end{array}$ & $\begin{array}{l}-0,001 \\
(0,003)\end{array}$ & $\begin{array}{c}-0,003 \\
(0,004)\end{array}$ & $\begin{array}{c}0,006 \\
(0,003)\end{array}$ & $\begin{array}{c}0,005 \\
(0,003)\end{array}$ \\
\hline $\begin{array}{c}c_{3} \\
\text { quarta }\end{array}$ & $\begin{array}{c}0,001 \\
(0,006)\end{array}$ & $\begin{array}{c}0,003 \\
(0,004)\end{array}$ & $\begin{array}{c}-0,001 \\
(0,002)\end{array}$ & $\begin{array}{c}0,001 \\
(0,003)\end{array}$ & $\begin{array}{r}-0,002 \\
(0,003)\end{array}$ & $\begin{array}{r}-0,004 \\
(0,003)\end{array}$ & $\begin{array}{r}-0,003 \\
(0,004)\end{array}$ & $\begin{array}{r}-0,004 \\
(0,003)\end{array}$ & $\begin{array}{c}0,004 \\
(0,004)\end{array}$ & $\begin{array}{c}0,000 \\
(0,003)\end{array}$ & $\begin{array}{r}-0,002 \\
(0,003)\end{array}$ \\
\hline $\begin{array}{c}c_{4} \\
\text { quinta }\end{array}$ & $\begin{array}{c}0,001 \\
(0,006)\end{array}$ & $\begin{array}{c}0,002 \\
(0,004)\end{array}$ & $\begin{array}{c}0,003 \\
(0,002)\end{array}$ & $\begin{array}{c}0,001 \\
(0,003)\end{array}$ & $\begin{array}{c}-0,003 \\
(0,004)\end{array}$ & $\begin{array}{l}-0,000 \\
(0,004)\end{array}$ & $\begin{array}{c}0,005 \\
(0,003)\end{array}$ & $\begin{array}{c}-0,000 \\
(0,004)\end{array}$ & $\begin{array}{c}-0,004 \\
(0,003)\end{array}$ & $\begin{array}{c}0,005 \\
(0,003)\end{array}$ & $\begin{array}{l}-0,002 \\
(0,003)\end{array}$ \\
\hline $\begin{array}{c}c_{5} \\
\text { sexta }\end{array}$ & $\begin{array}{l}-0,001 \\
(0,006)\end{array}$ & $\begin{array}{c}0,001 \\
(0,004)\end{array}$ & $\begin{array}{c}0,002 \\
(0,002)\end{array}$ & $\begin{array}{c}0,003 \\
(0,003)\end{array}$ & $\begin{array}{c}0,003 \\
(0,003)\end{array}$ & $\begin{array}{l}-0,002 \\
(0,003)\end{array}$ & $\begin{array}{l}-0,000 \\
(0,003)\end{array}$ & $\begin{array}{c}-0,002 \\
(0,003)\end{array}$ & $\begin{array}{c}0,000 \\
(0,004)\end{array}$ & $\begin{array}{c}0,003 \\
(0,003)\end{array}$ & $\begin{array}{c}0,007 \\
(0,003)\end{array}$ \\
\hline$\lambda$ & $\begin{array}{c}0,829 \\
(0,070)\end{array}$ & $\begin{array}{c}0,781 \\
(0,054)\end{array}$ & $\begin{array}{c}0,819 \\
(0,053)\end{array}$ & $\begin{array}{c}0,763 \\
(0,052)\end{array}$ & $\begin{array}{c}0,817 \\
(0,046)\end{array}$ & $\begin{array}{c}0,974 \\
(0,050)\end{array}$ & $\begin{array}{c}0,840 \\
(0,080)\end{array}$ & $\begin{array}{c}0,974 \\
(0,050)\end{array}$ & $\begin{array}{c}0,894 \\
(0,045)\end{array}$ & $\begin{array}{c}0,912 \\
(0,039)\end{array}$ & $\begin{array}{c}0,875 \\
(0,045)\end{array}$ \\
\hline DIC & -679 & -1014 & -1390 & -1154 & -1007 & -1102 & -1142 & -1102 & -1082 & -1226 & -1216 \\
\hline MSE & 1,495 & 0,006 & 0,004 & 0,049 & 0,006 & 0,005 & 0,005 & 0,005 & 0,005 & 0,005 & 0,004 \\
\hline
\end{tabular}

Tabela 2 - Resultados modelo 4 - subamostras anuais

Além dos resultados mencionados anteriormente foram obtidos, a partir de prioris vagas, as médias das posterioris dos parâmetros do modelo 4, para o índice Ibovespa com 15 mil 
iterações descartadas as 5 mil primeiras, e os resultados estão listados na tabela 1. Pode se observar que os resultados obtidos, com a estimação do modelo 4, não apresentam evidências para se admitir que exista sazonalidade diária no mercado brasileiro de ações no período estudado. Quanto ao número de graus de liberdade da distribuição t para a simulação realizada ficou em 5 .

Pode-se observar, que dentre os modelos apresentados na tabela 1, o modelo 4 apresentou a melhor performance quando o critério DIC (Deviance Information Criterion) é utilizado para seleção de modelos. O modelo 4 apresentou maior valor para o DIC. Acreditando ser esse critério o mais adequado, a partir dos resultados aqui descritos selecionou-se o modelo 4 para verificar o efeito dia da semana em amostras com os retornos dos índices de lucratividade dos anos: de 1994 até o ano de 2004, para o Ibovespa, perfazendo 11 amostras. Com isto pode-se inferir se a anomalia estudada tem variado no tempo no mercado brasileiro.

Os resultados com subamostras do Ibovespa, com 10 mil iterações descartadas as 5 mil primeiras, estão descritos na tabela 2 a seguir, com informações que da média da posteriori de cada parâmetro com o respectivo desvio padrão entre parênteses. Da observação da tabela 2, através das médias e desvios padrões das variáveis binárias que representam os dias da semana, verifica-se que em algumas amostras alguns dias apresentam significância, como por exemplo: em 1997 a hipótese de retornos diferenciados nas terças deve ser investigada mais detalhadamente. O mesmo acontece na quinta-feira na amostra do ano 2000, na segunda-feira do ano 2002, na terça-feira do ano de 2003, e na amostra do ano de 2004 na terça e na sexta. No entanto não existe um padrão que possa ser generalizado, isto é, não existem evidências de uma anomalia persistente, ou sistemática, em todas as amostra: seja para o efeito fim de semana ou dia da semana. Deve-se destacar, outrossim, que diferente do que aconteceu para outras amostras o parâmetro do modelo $\mathrm{AR}(1)$ para amostra do ano de 1998 não é significativo.

\section{Comentários finais}

Neste trabalho pode-se inferir o efeito fim de semana e dia da semana por intermédio de modelos autoregressivos heterocedásticos e verificar a existência da ocorrência de retornos diferenciados em algum dia da semana de forma sistemática, utilizando um modelo aqui selecionado para ser implementado em amostras anuais do índice Ibovespa. Os resultados não permitem aceitar a hipótese de retornos diferenciados em dias da semana, o que indica que o mercado apresenta uma alta eficiência no período estudado. Este resultado é compatível com crescimento e maior abertura do mercado de capitais brasileiro e uma maior estabilidade da economia desde 1994. No entanto outras pesquisas para uma maior investigação das hipóteses aqui verificadas.

As informações aqui utilizadas ficaram restritas a um índice de ações. Embora o Ibovespa seja um índice representativo do mercado de ações brasileiro, em pesquisas com o objetivo de verificar anomalias no mercado de ações devem incluir, além de outros índices, amostras de séries de retornos de ações individuais, pois as inferências podem fugir da realidade dos mercados por influência da metodologia de construção de determinado índice de lucratividade de ações. Dando continuidade a esta pesquisa, em um momento seguinte, serão analisadas séries de retornos das principais ações negociadas no mercado brasileiro observando-se os setores as quais estão vinculadas de forma a manter uma amostra diversificada, quanto aos setores da economia brasileira. Embora o modelo autoregressivo com heterocedasticidade tenha apresentado bons resultados para ajuste dos retornos do mercado acionário brasileiro, deve-se testar outros modelos autoregressivos heterocedásticos variantes.

Outro importante tópico a ser tratado e a variação sistemática do risco nos dias da semana. Deve-se destacar, outrossim, que a procura de um modelo que melhor se ajuste a séries de 
retornos de ativos financeiros, para investigação de variação sistemática dos retornos e da volatilidade, deve ser ampliada, principalmente no que tange ao tratamento da heterocedasticidade e com a implementação de modelos com distribuições de probabilidade alternativas para os retornos.

\section{Referências bibliográficas}

AGGARWAL, R., RIVOLI, P. (1989). Seasonal and Day-of-the-Week Effects in Four Emerging Stock Markets. The Financial Review, v. 24, pp. 541-550.

AGRAWAL, A., TANDON, K. (1994). Anomalies or illusions? Evidence from Stock Markets in Eighteen Countries. Journal of International Money and Finance, Vol. 13, No. 1, pp. 83-106.

BASHER, S., SADORSKY, P. (2005). Day-of-the-Week Effects in Emerging Stock Markets. in http://econpapers.repec.org (consulta em março de 2005).

BROOKS, C., PERSAND, G. (2001). Seasonality in Southeast Asian Stock Markets: Some New Evidence on Day-of-the-Week Effects. Applied Economics Letters, v. 8, pp. 155-158.

CROSS, F. (1973). The Behavior of Stock prices on Fridays and Mondays. Financial Analysts Journal, v. 29, pp. 67-69.

FRENCH, K. R. (1980). Stock Returns and The Weekend Effect, Journal of Financial Economics. v. 8, pp. 55-69.

GAMERMAN, D. (1997). Markov Chain Monte Carlo: Stochastic Simulation for Bayesian Inference. London, Chapman \& Hall.

GIBBONS, M., HESS, P. (1981). Day of the Week Effects and Asset Returns. Journal of Business, v. 54, pp. 579-596.

GILKS, W. R., RICHARDSON, S., SPIERGELHALTER, D. J. (1998). Markov Chain Monte Carlo in Practice. Boca Raton, Chapman \& Hall /CRC.

KEIM, D. B., STAMBAUGH, F. (1984). A Further Investigation of Weekend Effects in Stock Returns. Journal of Business, v. 39, pp. 819-889.

KOHERS, G., KOHERS, N., PANDEY, V., KOHERS, T. (2004). The Disappearing Day-of-the-Week Effect in the World's Largest Equity Markets. Applied Economics Letters, v. 11, pp. 167-171.

MIGON, H. S., GAMERMAN, D. (1999). Statistical Inference: An Integrated Approach. New York, Arnold Publishers.

PEIRÓ, A. (1994). The Distribution of Stock Returns: International Evidence. Applied Financial Economics, v. 4, pp. 431-439.

SPIERGELHALTER, D., BEST, N., CARLIN, B., LINDE, A. (2002). Bayesian Measures of Model Complexity and Fit. Journal of the Royal Statistical Society, Series B, v. 64, pp. 583-639.

SPIERGELHALTER, D., THOMAS, A., BEST, N. (2003). User Manual WinBUGS: version 1.3. MRC Biostatistics Unit. Institute of Public Health. Cambridge. UK.

TORRES, R., BONOMO, M., FERNADES, C. (2000). A Aleatoriedade do Passeio na Bovespa: Testando a Eficiência do Mercado Acionário Brasileiro. Ensaios Econômicos EPGE 402, Fundação Getúlio Vargas, Rio de Janeiro. 